Stanford Linesr Accelerator Center

Stanford Universit,y, Stanford, CA. 94305

\title{
INTRODUCIION
}

Th1s note describes the 2 Word $I / O$ subsystem and detalls 1 ts operating character1stics. SLAC drawngs in the 926-306 series support this device and should be referenced for construction and connection detalls.

GENERAL DESCRIPTION:

The 2 hord I/O subsystem consists of a single width CAMAC module, a sultch box, a display box and two interconnecting cables. The subsystem was developed for use on the SIAC 40" HBC Hybrid Facility (SHF) to provide insight into the data aquisition computer's operation during its data gathering operations. As the name implies the subsystem can handle two (2) 16 bit words in and twe (2) 16 bit words out. The 2 input words are controlled by 32 irddividual toggle switches which are sted onto the CAMAC dataway when approprlate CAMAC commands occur. Conversely, when the appropriate CAMAC commands appear on the CAMAC dataway, data is strobed, $16 \mathrm{~b} 1 \mathrm{is}$ at a time, into latches on the CAMAC nodule. These 32 latches drive 32 indicators in a remote Indicator box. Four of these latches have been wired to 50 ohm drivers whoso outputs are avallable on the front of the CAMAC module. In addition one of these latches enables on alarm circuit.

\section{COMMANDS AVAILABLE:}

The Following Commands Have Been Implemented:

$F(\emptyset) \cdot A(\emptyset)$.....Gates switch position information from word $\emptyset$ onto dataway $F(\sigma) \cdot A(1)$..... Gates switch position information from word 1 onto dataway $F(8) \ldots \ldots$....... Gates LAM ontc $Q$ bus of dataway

$F(10) \ldots \ldots$..... Hesets ILAM

* Work supported by the Depertment of Energy under contract number DE-:.03-76SF00515. 
$F(16) \cdot A(\phi) . .$. Takes first 16 bits off dataway urite lines and strobes them Into word $\square$

$F(16) \cdot A(1)$... Takes f1rst 16 b1ts off dataway wrjte lines and strobes them into word 1

$F(16) \cdot A(2)$... Takes first 4 b1ts off dataway write lines and strcbes them into tone reglster

$F(24) \ldots \ldots$. Resets ENABLE flip-flop

$F(26) \ldots \ldots$. Sets ENABLE flip-flop

$X$ is asserted for all executable instructions. $Q$ is asserted only on $F(\emptyset) \cdot A(\theta-14)-$ and $F(B)$ if LAM is on.

OPERATION DETAILS:

$F(\theta)$ Operation:

Issuing $F(\emptyset)$ results in dataway reads with the following conditions, depending on on the sub-address being used.

$\begin{array}{lccc}\text { Function } & \text { Data } & \underline{Q} & \underline{X} \\ F(\theta) \cdot A(\theta) & \text { 1st 16 sw1tohes (wd } \theta) & 1 & 1 \\ F(\theta) \cdot A(1) & \text { 2nd 16 switches (wd 1) } & 1 & 1 \\ F(\emptyset) \cdot A(2-14) & \text { All Zeros } & 1 & \emptyset \\ F(\emptyset) \cdot A(15) & \text { All Zeros } & \emptyset & \emptyset\end{array}$

Th1s Q characteristic allows Block transfer operation testing with some CAMAC crate controllers.

IAM OPERATION:

Normally, LAM will be set by an operator pushing a button on the remote box. If (or when) ENABLE is set IAM w1ll cause I to be asserted on the CAMAC dataway. If the CAMAC controller allows, the $\mathrm{L}$ w1ll cause an interivot to the host conputer. The host computer will respond by reading the contents of the 32 sultches. When the $F(\emptyset)$. A(1) occurs LAM will be reset.

If the programmer does not want to use interrupts he can disallow interruñs in the CAMAC controller and poll the controller for assertion of the $\mathrm{L}$ line.

If the programer does not want to use the $I$ line he can keep the ENABLE b1t in the HESET condition and poll the IAM statis by issuing an $F(B)$.

If the progremmer does not w13h to read the contents of the switches he can abort the cycle by 1ssuing an $F(10)$. 
$F(16) \cdot A(\emptyset)$ and $F(16) \cdot A(1)$ Operation:

32 Individual IED Indicators are always connected to the 32 bits in word $\emptyset$ and word 1. The elght least signiflcant bits (LSB) of word 1 are also connected to a dual hexidectmal display for added conventence.

The LSB of word 1 is also used as the tone enable bit for the alarm c1rcuit. Bits 2 (next to the LSB) and 16 of both words are also routed through 50 ohm coax drivers and are avallable on the front panel of the module on LEMO connectors. An assention 1s represented by a low level $(\emptyset .9-+\emptyset .5 \mathrm{v})$ and the negation by a high level $(+2.0 \mathrm{v}$ nomtnal).

Alarm Clrcuit Operation:

The tone generator is the basis for two-tone alarm system. For the alarm to sound, both the tone engble b1t (see above) and the ARM flip-flop must be set. The ARM flip-flop normally comes up in the RESET condition, 1t is set whenever output word 1 is accessed. The ARM flip-flop will be RESET if the operator pushes the SILENCE momentary pushbutton on the remote display box. Note that in this condition the tone enable bit is still sic so a visual indication of the ajarm condition is st1ll present.

The first four bits of sub address 2 form the tone generator word. The size of this word determines the differance between the base nate (a h1gh tone) and the alternate note (a lower tone). i.e. nots), 1111 cause maximum difference (a high note alternating w1th a low note). The length of each tone 1s fixed at approximately one quarter second.

The loudness of the alarm is fixed.

The loud speaker for the alarm 1s located in the remote display box. 